\title{
OBJETIVOS DE DESARROLLO SOSTENIBLE. RELEVAMIENTO INICIAL SOBRE LAS ACCIONES Y PROPUESTAS DE CONTRIBUCIÓN DE LAS FACULTADES DE CIENCIAS ECONÓMICAS NACIONALES ARGENTINAS A LA AGENDA 2030
}

\author{
Cavallo, Marcela Analía \\ Ledesma, Alicia Beatriz \\ Díaz, Liliana Patricia \\ Facco, Silvina María \\ Benzi, Carolina Soledad \\ Schmidt Strano, Erika
}

\section{RESUMEN:}

Este trabajo se realiza en el marco del proyecto de investigación "Aportes de la Universidad a la Agenda 2030. El impacto de las percepciones y expectativas de la comunidad académica de la Facultad de Ciencias Económicas y Estadística (UNR) en la consecución de los Objetivos de Desarrollo Sostenible" y presenta un relevamiento de acciones y propuestas de actividades y espacios en las facultades de ciencias económicas nacionales con relación a la propuesta de la Agenda 2030. En primer lugar, se muestran algunos aportes teóricos que han sido pilares para el diseño metodológico. A continuación, el estudio muestra la sistematización de la información disponible en los sitios web oficiales de las unidades académicas objeto de estudio analizada conforme a categorías teóricas desarrolladas en estudios tomados como referencia, que forman parte del estado del arte en el proyecto de investigación en curso. Como resultado del relevamiento exploratorio de los espacios virtuales de las unidades académicas en ciencias económicas nacionales argentinas, los primeros hallazgos permitieron además la construcción de categorías fundamentadas en esos datos que se incluyeron como subcategorías nuevas en los resultados. Finalmente fue posible arribar a conclusiones en torno a la propuesta teórica de Kestin et al (2017) y a las subcategorías definidas en este estudio, así como el grado de abordaje de las mismas para, finalmente, proponer algunas reflexiones acerca del grado de avance de la temática en Argentina.

PALABRAS CLAVE: Agenda 2030; Facultades Nacionales en Ciencias Económicas; Acciones y propuestas

\section{ABSTRACT:}

This investigation is carried out within the framework of the research project "Contributions of the University to the 2030 Agenda. The impact of the perceptions and expectations of the academic community of the Faculty of Economic Sciences and Statistics (UNR) in the 
achievement of the Sustainable Development Goals" and presents a survey of actions and proposals for activities and spaces in the faculties of national economic sciences in relation to the 2030 Agenda proposal. First, some theoretical contributions, that have been pillars for the methodological design, are shown. Next, the study shows the systematization of the information available on the official websites of the academic units under study analyzed according to theoretical categories developed in studies taken as reference, which are part of the state of the art in the current research project. As a result of the exploratory survey of the virtual spaces of the academic units in Argentine national economic sciences, the first findings also allowed the construction of categories based on these data that were included as new subcategories in the results. Finally, it was possible to reach conclusions about the theoretical proposal of Kestin et al (2017) and the subcategories defined in this study, as well as the degree of approach to them to, finally, propose some reflections about the degree of advance of the topic in Argentina.

KEY WORDS: Agenda 2030; National Faculties in Economic Sciences; Actions and proposals

\section{INTRODUCCIÓN.}

\section{UNIVERSIDADES, RESPONSABILIDAD SOCIAL UNIVERSITARIA (RSU) Y AGENDA 2030}

El rol de la universidad en la sociedad asume hoy nuevos retos que exceden a los tradicionales, ligados principalmente a aspectos académicos y de investigación. En los últimos años, estos debates se han potenciado en función de dos hitos relevantes.

Por un lado, la noción de RSU que adquiere renovadas fuerzas a comienzos de los años 2000 a través del trabajo conjunto entre la Red chilena "Universidad Construye País" y de la Red Latinoamericana de Universidades animada por la "Iniciativa Interamericana de Ética, Capital Social y Desarrollo". Y por otro, el impulso de la Conferencia Mundial de la UNESCO del 2009 que validó los intereses de universitarios al introducir la sostenibilidad a cuestiones ligadas a la investigación, la gestión y las relaciones con la sociedad. En la misma, la educación superior en tanto bien público, se declara competencia de las partes interesadas y de los gobiernos. Es además responsable de potenciar la comprensión de problemas considerando dimensiones sociales, económicas, científicas y culturales, asumir el liderazgo social en la creación de conocimientos, fomentar los aspectos interdisciplinarios y promover el pensamiento crítico y la ciudadanía activa, contribuyendo así al desarrollo sostenible, la paz y el bienestar, y materializar los derechos humanos.

La RSU supone un diálogo con la sociedad y una escucha activa de sus agentes (Vallaeys, 2014) que plantea una construcción basada en la gestión de los cuatro impactos que generan las instituciones de educación superior (Vallaeys et al. 2009) en relación con: 
- La organización misma, desde su campus y su personal: impactos laborales y medioambientales: gestión;

- La formación que imparte hacia los estudiantes: formación;

- Los conocimientos que construye desde sus centros de investigación y sus presupuestos epistemológicos, subyacentes a sus decisiones académicas: investigación,

- Sus relaciones con el entorno social, sus redes, contrataciones, relaciones de extensión, participaciones sociales, económicas y políticas, anclaje territorial: extensión.

Posteriormente Vallaeys avanza en su propuesta y en 2018 presenta el modelo URSULA, que trabaja idénticos ejes: extensión, formación, investigación y gestión. El avance lo da la sinergia que plantea entre los cuatro ejes al decir que la extensión de manera aislada y extracurricular no genera los impactos necesarios, sino que debe contar con la fuerza de la formación y el expertise de los investigadores. A su vez, la formación debe incluir en las currículas las cuestiones sociales y comunitarias y todo ello confluye en la necesidad de una investigación que permita innovar y contribuir con los ODS. Finalmente, esta retroalimentación es posible con una gestión socialmente responsable basada en un liderazgo responsable llevado adelante en su zona de influencia y con las alianzas necesarias

Otro de los hitos es el que refiere a la Agenda 2030. En el marco de la $70^{\circ}$ Cumbre del Desarrollo Sostenible (Naciones Unidas, 2015), se aprueba el documento "Transformando nuestro mundo: la Agenda 2030 para el Desarrollo Sostenible" convirtiéndose éste, en el acuerdo global más ambicioso de Naciones Unidas de los últimos años. Este documento toma como base los 17 problemas más urgentes y los convierte en un reto a resolver a través de la consecución de 17 objetivos y sus 169 metas, entre los que se incluyen la erradicación de la pobreza y el hambre; la protección del planeta de la degradación ambiental abordando el cambio climático; el asegurar que todas las personas puedan disfrutar vidas prósperas, saludables y satisfactorias; el fomentar sociedades pacíficas, justas e inclusivas, libres de violencia y sin miedo. Si bien esta Agenda no es legalmente vinculante y dado que ha sido elaborada con la metodología bottom-up, la acogida tanto de los gobiernos como de la sociedad civil y las empresas ha sido muy importante. En este escenario cobra relevancia el rol crítico de la universidad y la necesidad de su involucramiento.

En sintonía con lo expresado, Kestin et al (2017) elaboraron una propuesta que aúna los desarrollos anteriores (Fig. 1) 
Figura 1. ODS y Ejes de trabajo en las Universidades
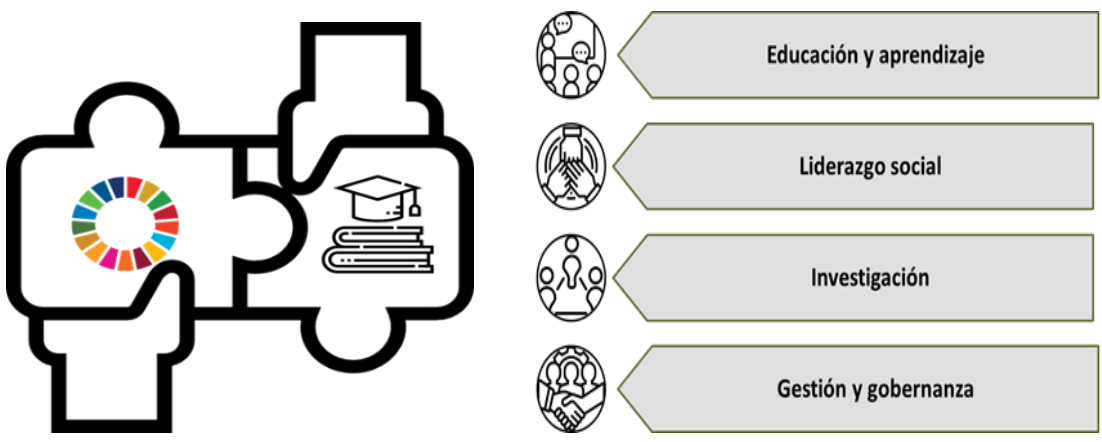

Elaboración propia en base a Kestin et al, 2017

Estos autores agrupan las acciones que llevan adelante las universidades, conforme a 4 ejes:

Educación y aprendizaje (Kestin et al, 2017, pág. 12)

- Dotar al alumnado de conocimientos, habilidades y motivación para entender y abordar los ODS

- Empoderar y movilizar a la juventud

- Proporcionar amplia formación académica o vocacional para implementar soluciones de ODS

- Crear más oportunidades para la creación de capacidades de estudiantes y profesionales de países en desarrollo para abordar los desafíos relacionados con los ODS

Liderazgo social (Kestin et al, 2017, pág. 28)

- Fortalecer el compromiso público y su participación pública en el ámbito de los ODS

- Iniciar y facilitar el diálogo, así como la acción intersectorial sobre la implementación de los ODS.

- Desempeñar un papel protagonista en el desarrollo y promoción de políticas de desarrollo sostenible

- Demostrar la importancia del sector universitario en la implementación de los ODS.

- Demostrar el compromiso del sector universitario con los ODS

Investigación (Kestin et al, 2017, pág. 18)

- Fomentar y promover los ODS como un tema de investigación dentro de la universidad

- Apoyar todo el espectro de enfoques de investigación necesarios para abordar los ODS, incluida la investigación interdisciplinar y transdisciplinar

- Apoyar y fomentar la innovación para soluciones de desarrollo sostenible

- Apoyar activamente la implementación nacional y local de los ODS

- Abogar por el apoyo nacional y la coordinación de la investigación sobre los ODS 
- Apoyar la creación de capacidades para que los países emergentes desarrollen y utilicen la investigación sobre los ODS.

Gestión y gobernanza (Kestin et al, 2017, pág. 23)

- Alinear las estructuras de gobierno universitario y las políticas operativas con los ODS

En una línea de investigación similar en Argentina, un estudio reciente (FSS, 2019) pone el acento en articular una estrategia con las universidades argentinas públicas y privadas para la difusión e implementación de los Objetivos de Desarrollo Sostenible (ODS) 2030. Entre sus principales hallazgos resalta la sistematización de acciones relacionadas con el tema.

\section{OBJETIVO}

Este escrito es una primera aproximación conceptual al conocimiento de las acciones y propuestas que llevan adelante las unidades académicas nacionales en ciencias económicas en Argentina y constituye una primera etapa de esta investigación. Se trata por tanto de un estudio exploratorio en estas instituciones con relación a su aporte al logro de los objetivos de desarrollo sostenible (ODS). Como toda investigación de carácter exploratorio, aplica una lógica que prioriza la extensión sobre la profundidad con el propósito de trazar un mapa de futuras etapas de investigación. En función de estas premisas este estudio propone:

- Analizar y sistematizar, en las unidades académicas objeto de estudio, las propuestas/acciones vinculadas a los ODS en función de los ejes: educación y aprendizaje- liderazgo social- investigación y gestión y gobernanza.

- Determinar los grados de abordaje generales de las acciones y propuestas en torno a los cuatro ejes estudiados.

\section{METODOLOGÍA}

Este trabajo se encuadra en la investigación cualitativa. En este camino y conforme al objetivo propuesto, el abordaje metodológico consistió en el relevamiento de páginas web oficiales de las unidades académicas objeto de estudio, en función de las categorías propuestas por la SDSN en "Cómo empezar con los ODS en las universidades" (Kestin et al, 2017). Aun con las limitaciones que supone un estudio de estas características, que toma como fuente primaria a los sitios virtuales que construye cada unidad académica - con diferentes formatos, información sintetizada y diverso énfasis en las áreas relevadas- se optó por este tipo de abordaje atendiendo al objetivo planteado y a la edad de esta investigación. 
De esta manera, la decisión metodológica de recurrir a este tipo de relevamiento es consonante a una investigación exploratoria, tanto en relación con el objeto a examinar con respecto de la vacancia de estudios extendidos sobre el tema en Argentina.

El trabajo de indagación se realizó durante los meses de marzo, abril y mayo de 2021. Se seleccionaron para esta etapa a las facultades/universidades/departamentos de ciencias económicas nacionales incluidas en el documento del Consejo de Decanos de Facultades en Ciencias Económicas ${ }^{1}$ - C.O.D.E.C.E- excluyendo intencionalmente a la Facultad de Ciencias Económicas y Estadística (UNR) que será objeto de estudio específico en una futura etapa de investigación.

En una primera etapa, y conforme a lo expresado, la búsqueda estuvo orientada a sistematizar los hallazgos en torno a 4 ejes: educación y aprendizaje, liderazgo social, investigación y gestión y gobernanza (Kestin et al, 2017). Simultáneamente en cada eje, a partir de las acciones y propuestas así relevadas, se definieron nuevas categorías fundamentadas en los datos relevados.

Finalmente, los hallazgos se sistematizaron en tablas y gráficos en los que se señaló la presencia de acciones y propuestas agrupadas en categorías teóricas de partida y subcategorías definidas por el equipo de investigación.

\section{RESULTADOS}

En primer lugar, se presentan los hallazgos sistematizados en torno a los ejes mencionados y conforme a las acciones y propuestas definidas como nuevas categorías para este estudio, surgidas de la aplicación de la teoría fundamentada en los datos. Por tratarse de un relevamiento que toma como fuente primaria a lo publicado por las unidades académicas

\footnotetext{
${ }^{1}$ Las unidades académicas relevadas son, en el orden de prelación que aparecen en el documento mencionado: Facultad de Ciencias Económicas. Universidad de Buenos Aires (UBA); Facultad de Ciencias Económicas y Administración. Universidad Nacional de Catamarca (UNCA); Facultad de Ciencias Económicas. Universidad Nacional del Centro de la Provincia de Buenos Aires (UNICEN); Facultad de Economía y Administración. Universidad Nacional del Comahue (UNCOMA); Facultad de Ciencias Económicas. Universidad Nacional de Córdoba (UNC); Facultad de Ciencias Económicas. Universidad Nacional de Cuyo (UNCU); Facultad de Ciencias de la Administración. Universidad Nacional de Entre Ríos (UNER1); Facultad de Ciencias Económicas. Universidad Nacional de Entre Ríos (UNER2); Facultad de Administración, Economía y Negocios. Universidad Nacional de Formosa (UnaF); Facultad de Ciencias Económicas. Universidad Nacional de Jujuy (UNJU); Facultad de Ciencias Económicas. Universidad Nacional del Litoral (UNL); Departamento de ciencias sociales. Universidad Nacional de Luján (UNLU); Departamento de Ciencias Económicas. Universidad Nacional de La Matanza (UNLAM); Facultad de Ciencias Económicas y Jurídicas. Universidad Nacional de la Pampa (UNLPam); Facultad de ciencias económicas. Universidad Nacional de La Plata (UNLP); Escuela de Economía y Negocios. Universidad Nacional de San Martín (UNSAM); Facultad de Humanidades, Ciencias Sociales y de la Salud. Universidad Nacional de Santiago del Estero (UNSE); Departamento de Ciencias de la Administración. Departamento de Economía. Universidad Nacional del Sur (UNS); Facultad de Ciencias Económicas. Universidad Nacional de Tucumán (UNT); Facultad de Ciencias Económicas. Universidad Nacional de la Patagonia San Juan Bosco (UNP); Facultad de Ciencias Económicas. Universidad Nacional de Lomas de Zamora (UNLZ); Facultad de Ciencias Económicas. Universidad Nacional de Mar del Plata (UNDMP); Facultad de Ciencias Económicas. Universidad Nacional de Misiones (UnaM); Facultad de Ciencias Económicas. Universidad Nacional del Nordeste (UNNE); Facultad de Ciencias Económicas. Universidad Nacional de Río Cuarto (UNRC); Facultad de Ciencias Económicas, Jurídicas y Sociales. Universidad Nacional de Salta (UNaS); Facultad de Ciencias Sociales. Universidad Nacional de San Juan (UNSJ; Facultad de Ciencias Económicas, Jurídicas y Sociales. Universidad Nacional de San Luís (ex Facultad de Ingeniería y Ciencias Económico-Sociales)(USL)
} 
en sus espacios virtuales oficiales, en esta instancia de investigación no es posible vislumbrar el grado de cumplimiento de las mismas o la fase de implementación que transitan. En las tablas que a continuación se presentan, se señalan con " $X$ "las acciones 0 propuestas observadas en las fuentes relevadas, mientras que los espacios vacíos indican que no fue posible encontrar información disponible. Las unidades académicas relevadas se identificaron según las siglas de la universidad de pertenencia.

\section{Tabla 1 Eje: Educación y aprendizaje}

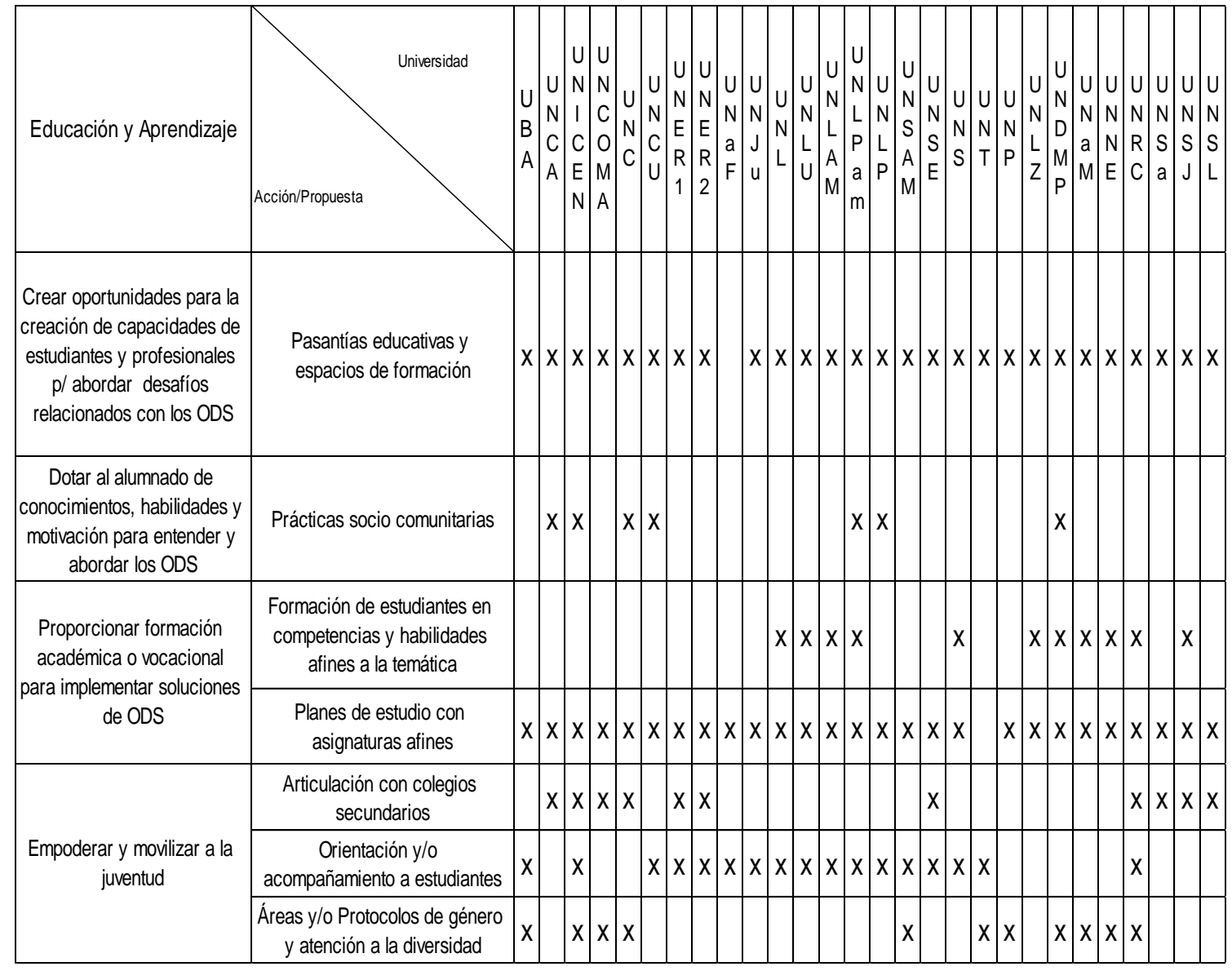

Fuente: elaboración propia 
Tabla 2 Eje: Liderazgo

\begin{tabular}{|c|c|c|c|c|c|c|c|c|c|c|c|c|c|c|c|c|c|c|c|c|c|c|c|c|c|c|c|c|}
\hline Liderazgo & + & $\begin{array}{l}\text { U } \\
B \\
\text { A }\end{array}$ & $\begin{array}{l}\mathrm{U} \\
\mathrm{N} \\
\mathrm{C} \\
\mathrm{A}\end{array}$ & $\begin{array}{l}\mathrm{U} \\
\mathrm{N} \\
\mathrm{I} \\
\mathrm{C} \\
\mathrm{E} \\
\mathrm{N}\end{array}$ & $\begin{array}{l}\text { U } \\
\text { N } \\
\text { C } \\
O \\
M \\
A\end{array}$ & $\begin{array}{l}\mathrm{U} \\
\mathrm{N} \\
\mathrm{C}\end{array}$ & $\begin{array}{l}\mathrm{U} \\
\mathrm{N} \\
\mathrm{C} \\
\mathrm{U}\end{array}$ & $\begin{array}{l}\mathrm{U} \\
\mathrm{N} \\
\mathrm{E} \\
\mathrm{R} \\
1\end{array}$ & $\begin{array}{l}\mathrm{U} \\
\mathrm{N} \\
\mathrm{E} \\
\mathrm{R} \\
2\end{array}$ & $\begin{array}{c}\mathrm{U} \\
\mathrm{N} \\
\mathrm{a} \\
\mathrm{F}\end{array}$ & $\begin{array}{c}\mathrm{U} \\
\mathrm{N} \\
\mathrm{J} \\
\mathrm{u}\end{array}$ & $\begin{array}{l}\mathrm{U} \\
\mathrm{N} \\
\mathrm{L}\end{array}$ & $\begin{array}{l}\mathrm{U} \\
\mathrm{N} \\
\mathrm{L} \\
\mathrm{U}\end{array}$ & \begin{tabular}{|c|}
$\mathrm{U}$ \\
$\mathrm{N}$ \\
$\mathrm{L}$ \\
$\mathrm{A}$ \\
$\mathrm{M}$
\end{tabular} & $\begin{array}{c}U \\
N \\
L \\
P \\
a \\
m\end{array}$ & 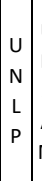 & \begin{tabular}{l|l}
$\mathrm{U}$ & \\
$\mathrm{N}$ & \\
$\mathrm{S}$ & \\
$\mathrm{A}$ & \\
$\mathrm{M}$ &
\end{tabular} & $\begin{array}{l}U \\
N \\
S \\
E\end{array}$ & & & $\begin{array}{l}U \\
N \\
P\end{array}$ & \begin{tabular}{l|l}
$U$ & $U$ \\
$N$ & $N$ \\
L & $D$ \\
$Z$ & $M$ \\
& $P$
\end{tabular} & $\begin{array}{l}\mathrm{U} \\
\mathrm{N} \\
\mathrm{a} \\
\mathrm{M}\end{array}$ & $\begin{array}{l}\mathrm{U} \\
\mathrm{N} \\
\mathrm{N} \\
\mathrm{E}\end{array}$ & $\begin{array}{l}\mathrm{U} \\
\mathrm{N} \\
\mathrm{R} \\
\mathrm{C}\end{array}$ & $\begin{array}{l}\mathrm{U} \\
\mathrm{N} \\
\mathrm{S} \\
\mathrm{a}\end{array}$ & $\begin{array}{l}\mathrm{U} \\
\mathrm{N} \\
\mathrm{S} \\
\mathrm{J}\end{array}$ & \\
\hline $\begin{array}{l}\text { Desempeñar un } \\
\text { papel protagonista } \\
\text { en el desarrollo y } \\
\text { promoción de } \\
\text { políticas de } \\
\text { desarrollo sostenible }\end{array}$ & $\begin{array}{l}\text { Vinculación y extensión en conjunto con } \\
\text { organizaciones sociales, gubernamentales y de } \\
\text { la sociedad civil, procurando concretar acciones } \\
\text { transformadoras. }\end{array}$ & $x$ & $\mathbf{x}$ & $x$ & $\mathbf{x}$ & $\mathbf{x}$ & $x$ & $\mathbf{x}$ & $\mathbf{x}$ & $\mathbf{x}$ & $\mathbf{x}$ & $x$ & $\mathbf{x}$ & $x$ & $\mathbf{x}$ & $\mathbf{x}$ & $x$ & \begin{tabular}{l|l}
$\mathbf{x}$ & $\mathbf{X}$
\end{tabular} & $x$ & 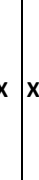 & $x \mid x$ & $\begin{array}{lll}x & x\end{array}$ & $\mathbf{x}$ & $x$ & $x$ & $x$ & $\mathbf{x}$ & $x$ \\
\hline \multirow{4}{*}{$\begin{array}{l}\text { Demostrar el } \\
\text { compromiso del } \\
\text { sector universitario } \\
\text { con los ODS }\end{array}$} & $\begin{array}{c}\text { Voluntariado y desarrollo del potencial solidario } \\
\text { para contribuir a la construcción del futuro } \\
\text { deseado para el bienestar general }\end{array}$ & $x$ & $\mathbf{x}$ & $x$ & & $x$ & $x$ & $x$ & & X & & $x$ & $x$ & & & $x$ & & & & $\mathrm{x}$ & $x$ & & & $x$ & & & & \\
\hline & $\begin{array}{l}\text { Orientación al Emprendedor externo para } \\
\text { desarrollar un conjunto de capacidades } \\
\text { relacionados con la toma de decisiones y el } \\
\text { trabajo autónomo.. }\end{array}$ & & & & & & & & $\mathbf{x}$ & $x$ & & X & & & & & & & $\mathrm{x}$ & & & & $x$ & $\mathbf{x}$ & & $x$ & $\mathbf{x}$ & $x$ \\
\hline & $\begin{array}{c}\text { Cátedras/Clases abiertas destinadas al público } \\
\text { en general para reflexionar sobre temas } \\
\text { relevantes vinculados a la economía social y } \\
\text { solidaria }\end{array}$ & $x$ & & & & & & & $x$ & & & & & & & $x$ & & $x$ & & & & & & & & & & \\
\hline & $\begin{array}{l}\text { Capacitación en temáticas sociales dirigidas al } \\
\text { público en general, para responder a las } \\
\text { necesidades de formación de los actores del } \\
\text { medio socio productivo. }\end{array}$ & $x$ & $\mathbf{x}$ & $x$ & $\mathbf{x}$ & $\mathbf{x}$ & $x$ & $x$ & $x$ & $\mathbf{x}$ & $\mathbf{x}$ & $\mathbf{x}$ & $\mathbf{x}$ & $\mathbf{x}$ & $x$ & $\mathbf{x}$ & $x$ & $x$ & $x$ & $\mathrm{x}$ & $x$ & $x$ & $x$ & $x$ & $\mathbf{x}$ & $x$ & $x$ & $\mathrm{x}$ \\
\hline
\end{tabular}

Fuente: elaboración propia

Tabla 3 Eje: Investigación

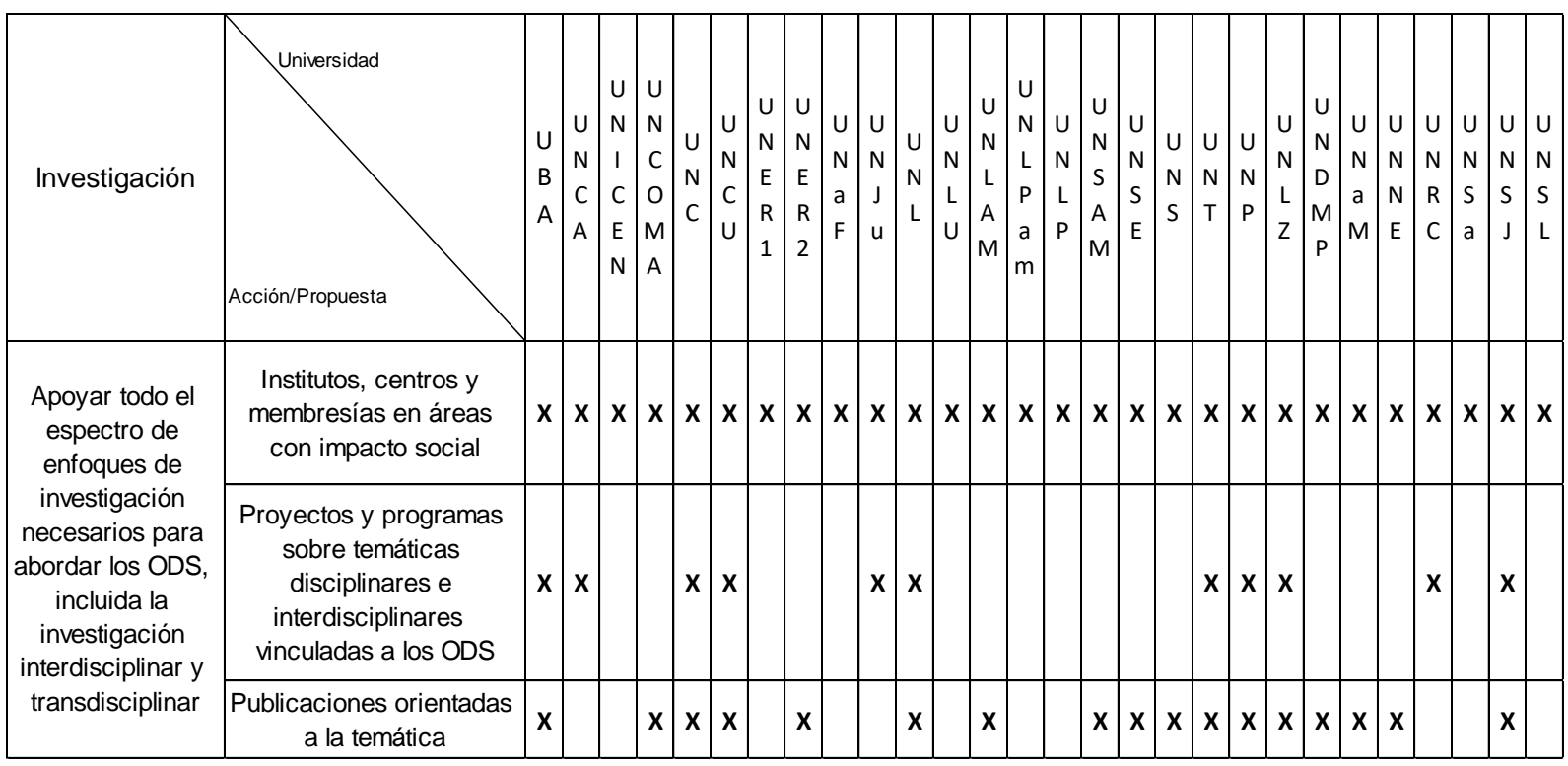

Fuente: elaboración propia 
Tabla 4 Eje: Gestión y Gobernanza

\begin{tabular}{|c|c|c|c|c|c|c|c|c|c|c|c|c|c|c|c|c|c|c|c|c|c|c|c|}
\hline $\begin{array}{l}\text { Gestión y } \\
\text { Gobernanza }\end{array}$ & Acción/Propuesta & $\begin{array}{l}U \\
B\end{array}$ & $\begin{array}{l}U \\
\mathrm{U} \\
\mathrm{C} \\
\mathrm{A}\end{array}$ & $\begin{array}{ccc}U & L \\
N & N \\
I & C \\
C & C \\
E & N \\
N & A\end{array}$ & \begin{tabular}{l|l}
$U$ & \\
$N$ & \\
$C$ & $U$ \\
$O$ & $N$ \\
$M$ & $O$ \\
$A$ &
\end{tabular} & $\begin{array}{l}\mathrm{U} \\
\mathrm{N} \\
\mathrm{C} \\
\mathrm{U}\end{array}$ & $\begin{array}{l}U \\
N \\
E \\
R \\
1\end{array}$ & $\mid \begin{array}{ll}U & \\
N & 1 \\
E & 1 \\
R & \\
2 & \end{array}$ & \begin{tabular}{l|l}
$U$ & $U$ \\
$N$ & $N$ \\
$a$ & $J$ \\
$F$ & $U$
\end{tabular} & $\begin{array}{l}\mathrm{U} \\
\mathrm{N} \\
\mathrm{L}\end{array}$ & \begin{tabular}{l|l}
$U$ & $U$ \\
$N$ & $N$ \\
$L$ & $L$ \\
$U$ & $A$ \\
$N$
\end{tabular} & \begin{tabular}{l|l}
$U$ & $U$ \\
$N$ & $N$ \\
$L$ & $L$ \\
$A$ & $P$ \\
$M$ & $a$ \\
$m$
\end{tabular} & $\begin{array}{l}U \\
N \\
L \\
P\end{array}$ & \begin{tabular}{l|l}
$U$ & $U$ \\
$N$ & $N$ \\
$S$ & $N$ \\
$A$ & $S$ \\
$M$ & $E$
\end{tabular} & \begin{tabular}{l|l}
$U$ & $U$ \\
$N$ & $N$ \\
$S$ & $S$
\end{tabular} & $\begin{array}{c}U \\
N \\
T\end{array}$ & \begin{tabular}{l|l}
$U$ & $U$ \\
$N$ & $N$ \\
$P$ & $L$ \\
$Z$
\end{tabular} & $\begin{array}{l}U \\
N \\
N \\
D \\
M \\
P\end{array} \mid$ & \begin{tabular}{l|l}
$U$ & $L$ \\
$N$ & $N$ \\
$a$ & $N$ \\
$M$ & $E$
\end{tabular} & \begin{tabular}{l|l}
$U$ & $U$ \\
$N$ & $N$ \\
$N$ & $R$ \\
$E$ & $C$
\end{tabular} & $\begin{array}{l}U \\
N \\
S \\
a\end{array}$ & $\begin{array}{l}U \\
N \\
S \\
J\end{array} \mid$ & $\begin{array}{l}\text { U } \\
N \\
\text { S } \\
\text { L }\end{array}$ \\
\hline $\begin{array}{l}\text { Alinear las } \\
\text { estructuras de } \\
\text { gobierno }\end{array}$ & $\begin{array}{l}\text { Declaración de misión, } \\
\text { visión y valores } \\
\text { vinculados a la } \\
\text { sostenibilidad y los ODS }\end{array}$ & $\mathrm{x}$ & & $\mathrm{x}$ & $x \mid x$ & $x|x|$ & $x$ & $x$ & \begin{tabular}{l|l}
$x$ & $x$
\end{tabular} & $x$ & \begin{tabular}{l|l}
$x$ & $x$
\end{tabular} & \begin{tabular}{l|l}
$x$ & $x$
\end{tabular} & $x$ & $x$ & \begin{tabular}{l|l}
$x$ & $x$
\end{tabular} & & \begin{tabular}{l|l}
$x$ & $x$
\end{tabular} & \begin{tabular}{l|l|}
$x$ & $x$
\end{tabular} & $x \mid x$ & $x \mid x$ & $x$ & $x$ & $x$ \\
\hline $\begin{array}{l}\text { universitario y las } \\
\text { políticas } \\
\text { operativas a los } \\
\text { ODS }\end{array}$ & $\begin{array}{c}\text { Protocolos, } \\
\text { procedimientos y } \\
\text { programas específicos } \\
\text { vinculados a la gestión } \\
\text { sostenible }\end{array}$ & $x$ & $\mathrm{x}$ & & $x$ & & & $\mathrm{x}$ & $\mathrm{x}$ & & $\mathrm{x}$ & $\mathrm{x}$ & & $x$ & \begin{tabular}{l|l}
$x$ & $x$
\end{tabular} & $x$ & & & & $x$ & & & \\
\hline
\end{tabular}

Fuente: elaboración propia

A continuación, interesa presentar los resultados agrupados en torno a las acciones y propuestas, indicando para cada una el porcentaje abordado para cada eje.

Gráfico 1. Abordaje de Acciones y propuestas vinculadas al eje: Educación y aprendizaje

Planes de estudio con asignaturas afines

Pasantías educativas y espacios de formación

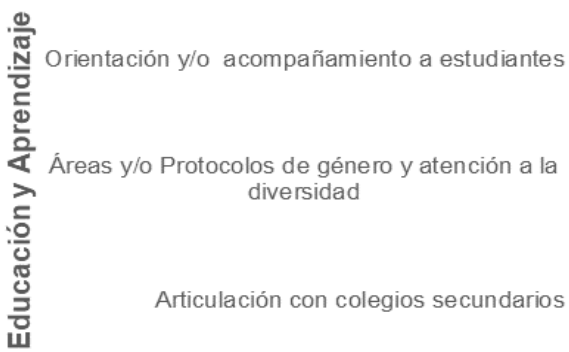

Formación de estudiantes en competencias y habilidades afines a la temática

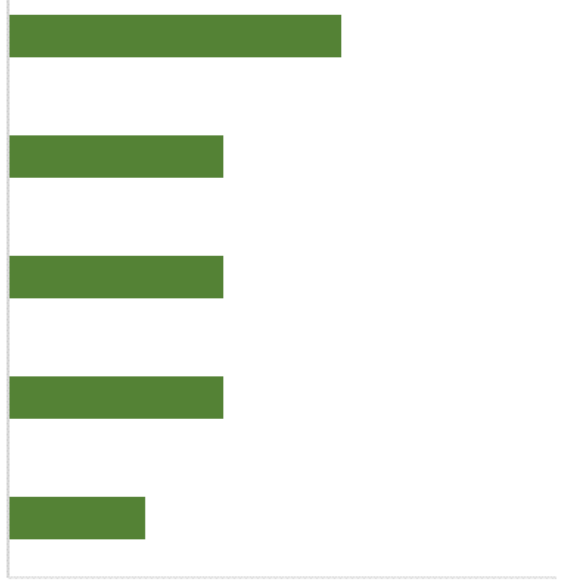

Fuente: Elaboración propia 
El gráfico 1 revela para este eje que los mayores porcentajes de acciones y propuestas se focalizan en los planes de estudio con asignaturas vinculadas a la temática (en forma indirecta) y en las pasantías educativas. Mientras que los menores \% se encuentran en las prácticas socio comunitarias.

\section{Gráfico 2. Abordaje de Acciones y propuestas vinculadas al eje: Liderazgo}

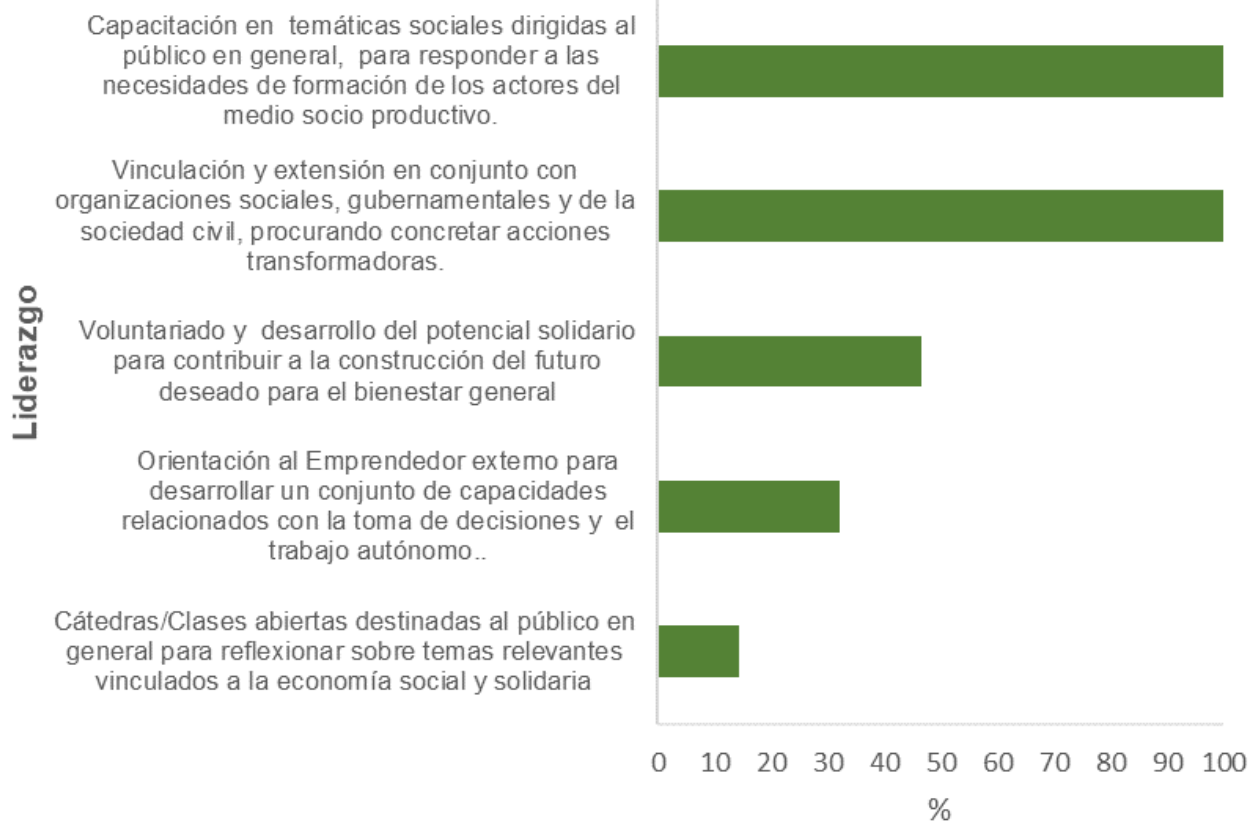

Fuente: Elaboración propia

En el gráfico 2, pueden observarse los mayores \% en la capacitación en temáticas sociales y en la vinculación y extensión; la cantidad de cátedras y/o clases abiertas destinadas al público sobre temas vinculados a la temática de este estudio, reúne el menor porcentaje.

\section{Gráfico 3. Abordaje de Acciones y propuestas vinculadas al eje: Investigación}

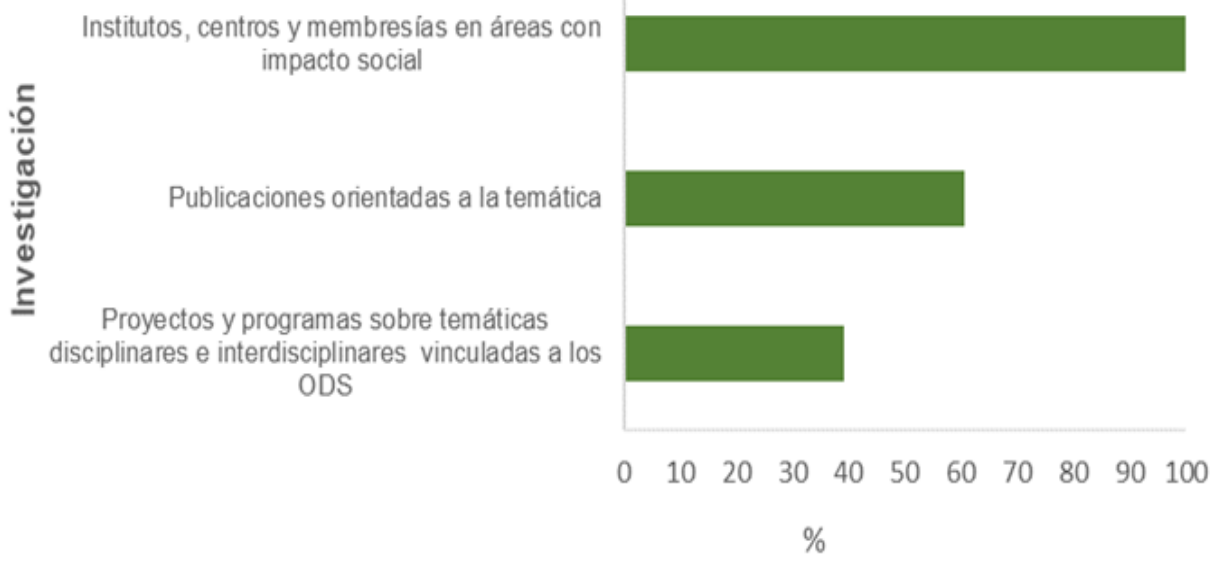

Fuente: Elaboración propia 
Para el eje investigación, el gráfico 3 revela hallazgos con mayor \% en lo referido a la declaración de misión, visión y valores relacionados a la temática de la sostenibilidad.

\section{Gráfico 4. Abordaje de Acciones y propuestas vinculadas al eje: Gestión y Gobernanza}

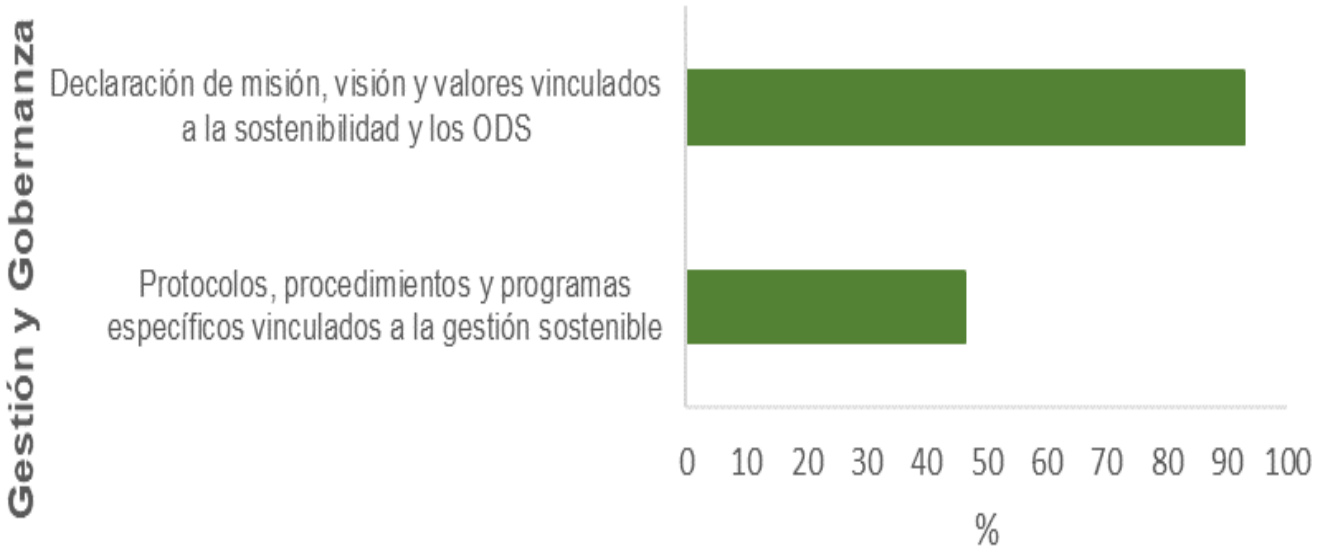

Fuente: Elaboración propia

El gráfico 4 muestra que los mayores \% en el eje gobernanza se sitúan en la existencia de institutos, centros y membresías relacionadas a áreas con impacto social.

\section{A MODO DE CIERRE}

Del análisis de las acciones y propuestas comunicadas por las unidades académicas en los sitios web relevados en este estudio fue posible arribar a algunas conclusiones de carácter preliminar.

En primer lugar, respecto del eje educación y aprendizaje las acciones y propuestas de las unidades académicas se concentran en "crear más oportunidades para la creación de capacidades de estudiantes y profesionales de países en desarrollo para abordar los desafíos relacionados con los ODS" a través de la oferta de pasantías conforme a la Ley 26427, alineadas con esta acción en la medida que las actividades propuestas en su marco sean consistentes a la Agenda 2030.

Respecto de "Dotar al alumnado de conocimientos, habilidades y motivación para entender y abordar los ODS" se vislumbra en algunas unidades académicas y se materializa a través de talleres, programas e iniciativas relacionadas a la ética, la responsabilidad social y el desarrollo de la cultura emprendedora.

Las propuestas ligadas a protocolos de género y articulaciones con escuelas medias presentes en la mayoría de las unidades académicas relevadas se relacionan con "Empoderar y movilizar a la juventud". 
Proporcionar amplia formación académica o vocacional para implementar soluciones de ODS se plasma levemente en los contenidos de los planes de estudio, en la mayoría de las unidades académicas por medio de materias electivas/optativas y en menor medida en asignaturas obligatorias. Se destaca que la Facultad de Ciencias Económicas - Universidad Nacional de la Patagonia "San Juan Bosco", cuenta con la carrera de Tecnicatura universitaria en administración ambiental.

Respecto del ítem "Crear más oportunidades para la creación de capacidades de estudiantes y profesionales de países en desarrollo para abordar los desafíos relacionados con los ODS" no se relevaron propuestas asociadas.

En segundo lugar, y en relación con el eje Liderazgo social resaltan las propuestas ligadas a Fortalecer el compromiso público y su participación pública en el ámbito de los ODS. Del relevamiento de las acciones surge la intencionalidad evidenciada en la integración de estrategias que pueden contribuir al cumplimiento de los 17 ODS y sus metas. Otra cuestión observada es la colaboración, forjando relaciones sólidas y transparentes con los grupos de interés, trabajando y desarrollando actividades.

En general las facultades de las universidades nacionales poseen rasgos de un liderazgo visionario, por el cual proyectan objetivos desafiantes, que motivan a la observación de determinadas cuestiones sociales, ambientales y económicas, como observatorios económicos, de comercio internacional, de derechos humanos, de PYME, y muchos otros. También se destacan Programas: ODS en movimientos, y procesos de desarrollo institucional y educativo que llevan a cabo internamente.

Hay relaciones positivas con la comunidad, por ejemplo, las actuaciones en extensión y voluntariado. La divulgación del quehacer cultural, las cátedras abiertas y además otras áreas problemáticas; consumos y prevención de adicciones, violencia de género, sobre gestión del agua, programas de adultos mayores, programas para mayores de 25 años sin secundaria, programas que vinculan a la Universidad y la escuela secundaria o a barrios locales, todos son ejemplos para resaltar. Algunas unidades académicas sobresalen por otorgar premios.

En referencia a Desempeñar un papel protagonista en el desarrollo y promoción de políticas de desarrollo sostenible se visibiliza su contribución con capacitaciones, simposios, encuentros virtuales, diplomaturas, y doble titulación.

Algunas de las facultades asumen la participación o involucramiento en relación a emprendedurismo, muestra de ello son: el gabinete de emprendedores, la oficina de orientación al emprendedor, incubadoras que acompañan a transitar el camino emprendedor, y emprendimientos con alumnos por medio de préstamos. 
En referencia al eje Investigación; las acciones y propuestas de las unidades académicas se concentran en "Apoyar todo el espectro de enfoques de investigación necesarios para abordar los ODS, incluida la investigación interdisciplinar y transdisciplinar - Apoyar y fomentar la innovación para soluciones de desarrollo sostenible". El relevamiento llevado a cabo, muestra de manera explícita la presencia de institutos, centros de estudios socioeconómicos, revistas de divulgación, régimen de becas, observatorios socio económicos, generación de plataformas colaborativas, como así también grupos de estudios ambientales que impulsan y promueven la investigación interdisciplinar y transdisciplinar. En algunas de las unidades académicas se señalan líneas de investigación ligadas a problemáticas vinculadas a la contribución del desarrollo de ciudades sustentables, proyectos de apoyo al emprendedurismo, a la pequeña y mediana empresa, al cooperativismo y fomento de economías regionales a través de polos ecológicos. También se señalan líneas de investigación relacionadas con cuestiones asociadas a la multiculturalidad e interculturalidad, como así también a la responsabilidad social empresaria, la ética administrativa, el balance social, las tendencias en el mundo del trabajo y su impacto en la creación de valor económico social- ambiental. Se muestran también líneas que refieren a políticas públicas y sociales con impacto territorial, vulnerabilidad social, pobreza, exclusión, cuestiones de género.

Por último, en referencia a la Gestión y gobernanza y respecto de "Alinear las estructuras de gobierno universitario y las políticas operativas con los ODS" el relevamiento muestra que algunas Facultades en forma expresa refieren a planes estratégicos, misión y visión enfocados en la formación de calidad, competencias y proyección social de las titulaciones. En menor número, algunas Facultades sólo señalan la estructura como organigrama o como una descripción de secretarías y departamentos.

Una limitación importante en el estudio fue la posibilidad de corroborar que las acciones y propuestas estén vigentes en la actualidad. No obstante, conforme al objetivo de investigación los resultados se presentan consistentes con un primer relevamiento de propuestas.

En general se observa que no existe mención expresa a los ODS para ninguno de los ejes trabajados en este escrito, a excepción de algunas unidades académicas que cuentan con proyectos de investigación y extensión específicos de la temática, en los que aparece la referencia literal.

Entre las acciones ligadas a los ODS, que no apelan directamente a esta terminología, se observa que en la gran mayoría de las unidades académicas relevadas se trabaja en políticas de género y cuentan con protocolos de actuación frente a situaciones de violencia y discriminación. También es notable la coincidencia en la aplicación de regímenes de pasantías. De todos modos, ambos tipos de acciones obedecen a cambios normativos. 
Siguiendo con la línea de acciones y propuestas vinculadas indirectamente a los ODS por no mencionarlos expresamente, se vislumbran algunas que obedecen a cambios culturales muy presentes en muchas unidades académicas, tales como las ligadas al emprendedurismo y en menor medida las vinculadas a contenidos curriculares en los planes de estudio de las carreras.

Cabe destacar que los hallazgos de este estudio no pretenden dar una respuesta acabada a la pregunta de investigación. Sin embargo, este primer relevamiento es un paso indispensable para permitir el mapeo de propuestas en las unidades académicas nacionales a profundizar en análisis posteriores.

Para concluir, se observa que las universidades nacionales argentinas han iniciado un proceso para trabajar en pos de las personas, el planeta, la prosperidad, la paz y las alianzas, pero el mismo se presenta de manera dispar en función de las características propias de la unidad académica, el espacio que ocupa, las diferencias de poder y sus relaciones.

Si bien se observa que no existe mención expresa a la Agenda 2030 en las acciones y propuestas relevadas para los ejes presentados, algunas de ellas cuentan con proyectos de investigación y extensión específicos sobre ODS y en cuanto a lo académico, se observan algunos contenidos curriculares afines y una variada oferta extracurricular.

\section{REFERENCIAS BIBLIOGRÁFICAS}

Foro del Sector Social - Federación - (FSS), la Asociación Argentina de Salud Pública (AASAP)y la Red Argentina de Instituciones Académicas en Apoyo a la Implementación de los Objetivos de Desarrollo Sostenible 2030 (REDAA-2030) (2019) Universidades Argentinas y los ODS, Posicionando la Agenda 2030 recuperado de http://forodelsectorsocial.org.ar/wp/wpcontent/uploads/2020/02/Universidades-y-ODS-Informe-Final-y-Anexos-12-2019.pdf.

Kestin, T.; Van den Belt,M; Denby, L.; Ross K.,Thwaites, J; Hawkes, M; (2017). "Cómo empezar con los ODS en las Universidades" Getting started with the SDGs in universities: A guide for universities, higher education institutions, and the academic sector. Australia, New Zealand and Pacific Edition. Sustainable Development Solutions Network - Australia/Pacific, Melbourne

https://www.utn.edu.ar/images/Secretarias/SGral/Integracion/GuiaUNiversidadesODS.pdf

Unión de Responsabilidad Social Universitaria Latinoamericana [URSULA] (2018). Investigación Continental URSULA: Estado del arte de la Responsabilidad Social Universitaria (RSU) en América Latina. Recuperado de: 


\section{FUENTES}

www.economicas.uba.ar

www.eco.unca.edu.ar

www.econ.unicen.edu.ar

www.faeaweb.uncoma.edu.ar/

www.eco.unc.edu.ar

www.fce.uncuyo.edu.ar

www.fcad.uner.edu.ar

www.fceco.uner.edu.ar

www.faen.unf.edu.ar

www.fce.unju.edu.ar

www.unsam.edu.ar

www.fhu.unse.edu.ar

www.uns.edu.ar/deptos/administracion

www.face.unt.edu.ar

www.unp.edu.ar

www.economicas.unlz.edu.ar

www.eco.mdp.edu.ar

www.fce.unam.edu.ar

www.unne.edu.ar

www.eco.unrc.edu.ar/seccion

www.economicas.unsa.edu.ar

www.facso.unsj.edu.ar

www.fcejs.unsl.edu.ar

www.fce.unl.edu.ar

www.cienciassociales.unlu.edu.ar/

www.economicas.unlam.edu.ar/

www.eco.unlpam.edu.ar/

www.econo.unlp.edu.ar 